\title{
SMR
}

\section{Development and characterization of microsatellite loci in Brasenia schreberi (Cabombaceae) based on the next-generation sequencing}

H. Liu' ${ }^{1 *}$, Z.C. Long ${ }^{2 *}$, L.N. Li' ${ }^{2}$, Q.F. Wang ${ }^{2}$, J.M. Chen ${ }^{2}$ and K. Liao ${ }^{2}$

${ }^{1}$ College of Life Science, South-Central University for Nationalities, Wuhan, Hubei, China

${ }^{2}$ Key Laboratory of Plant Germplasm Enhancement and Specialty Agriculture, Wuhan Botanical Garden, Chinese Academy of Sciences, Wuhan, Hubei, China

*These authors contributed equally to this study. Corresponding author: K. Liao

E-mail: liaokuo@wbgcas.cn

Genet. Mol. Res. 15 (2): gmr.15027886

Received Ouctober 22, 2015

Accepted December 22, 2015

Published April 4, 2016

DOI http://dx.doi.org/10.4238/gmr.15027886

ABSTRACT. To understand genetic variability of the endangered aquatic herb Brasenia schreberi (Cabombaceae), we describe 31 microsatellite markers obtained using next-generation sequencing. A total of 24 individuals from the population of Jackson Lake, USA, were genotyped for each marker. Twenty-eight markers were polymorphic. The number of alleles per locus ranged from 1 to 9; the observed and expected heterozygosities ranged from 0 to 1 and from 0 to 0.751 , respectively. These markers should be useful tools for genetic variation and conservation studies of $B$. schreberi.

Key words: Aquatic herb; Brasenia schreberi; Endangered species; Microsatellite marker; Next-generation sequencing 


\section{INTRODUCTION}

Brasenia schreberi J.F. Gmelin (Cabombaceae), a perennial floating-leaved aquatic herb (water shield), is distributed widely in temperate and tropical regions of Asia, Australia, Africa, India, and North and South America (Kim et al., 2008, 2012). However, populations of B. schreberi are found only rarely in each region. In several countries of East Asia, B. schreberi has been listed as a critically endangered species due to human activity and habitat loss (Lee et al., 2005; Zhang and Gao, 2008).

In recent years, genetic variation among $B$. schreberi populations has been demonstrated with a variety of dominant genetic markers, e.g., RAPD (random amplified polymorphic DNA) and AFLP (amplified fragment length polymorphism) (Kim et al., 2008), ISSR (inter-simple sequence repeat) (Zhang and Gao, 2008), and nuclear ribosomal ITS and chloroplast DNA trnL-F sequences (Kim et al., 2012). However, the results of these studies are still not sufficient for defining adaptive population differentiation. Next-generation high-throughput DNA sequencing techniques have greatly promoted the development of multiple co-dominant markers, such as simple sequence repeat (SSR) markers (Chen et al., 2015; Yun et al., 2015; Zhang et al., 2015), which have been shown to be useful tools for assessing the genetic diversity and adaptive population differentiation of endangered species. Here, we report the development of microsatellite markers from $B$. schreberi using next-generation sequencing, which will facilitate ongoing studies into the evolutionary history and conservation of this endangered species.

\section{MATERIAL AND METHODS}

Fresh leaves of an individual of $B$. schreberi plant were collected from Wuhan Botanical Garden, China, and genomic DNA was extracted using a Plant Genomic DNA Isolation kit (Tiangen, Beijing, China) following the manufacturer instructions. A sequencing library was constructed at Novogene Bioinformatics Technology Co., Ltd. (Beijing, China) according to the manufacturer instructions (Illumina). This library was sequenced using an Illumina HiSeq 2000 Platform at Novogene Bioinformatics Technology Co., Ltd., and generated $5.59 \mathrm{G}$ clean DNA reads. A total of 106,801 SSR loci were identified using the MIcroSAtellite identification tool (MISA) (Thiel et al., 2003) from the resources, and 54,640 primers were successfully designed using Primer3 (http:// biotools.umassmed.edu/bioapps/primer3_www.cgi).

Fifty of the designed primers were randomly selected and initially screened using total DNA isolated from dried leaves of six B. schreberi individuals collected from the population at Mangshan in Hunan Province. Polymerase chain reaction (PCR) amplifications were performed in a volume of $20 \mu \mathrm{L}$ containing $0.25 \mathrm{mM}$ each dNTP, $2 \mu \mathrm{L} 10 \mathrm{X}$ Taq buffer $(10 \mathrm{mM}$ Tris- $\mathrm{HCl}, \mathrm{pH} 8.3$, $1.5 \mathrm{mM} \mathrm{MgCl}_{2}$, and $50 \mathrm{mM} \mathrm{KCl}$ ), $1 \mathrm{mM}$ each primer, $0.2 \cup$ Taq Polymerase (TransGen Biotech Co., Beijing, China), and 25 ng DNA template. Genomic DNA was amplified using an ABI 2720 Thermal Cycler (Applied Biosystems, USA). The PCR program was as follows: an initial denaturation of 5 min at $94^{\circ} \mathrm{C}$, followed by 35 cycles of $30 \mathrm{~s}$ at $94^{\circ} \mathrm{C}, 30 \mathrm{~s}$ annealing at $54^{\circ}-56^{\circ} \mathrm{C}$ (depending on the primers), and $30 \mathrm{~s}$ extension at $72^{\circ} \mathrm{C}$, with a final extension cycle of 10 min at $72^{\circ} \mathrm{C}$. Agarose gel electrophoresis showed that 31 of 50 markers were clearly amplified.

Based on the initial screening results, all of the 31 primer pairs were selected and used to test for polymorphism in 24 individuals from a population located in Jackson Lake, Florida, USA $\left(85^{\circ} 11^{\prime} \mathrm{W}, 30^{\circ} 45^{\prime} \mathrm{N}\right)$. Forward primers were labeled with 6-FAM. PCR was performed using the same reaction and cycling conditions as were used in the initial screening. PCR products were separated using an $\mathrm{ABI} 3730 \mathrm{XL}$ automated sequencer (TsingKe Biotech, Beijing, China) and 
visualized using the GeneScan system (Applied Biosystems, Foster City, CA, USA). The number of observed alleles per locus $\left(N_{\mathrm{A}}\right)$, observed $\left(H_{\mathrm{O}}\right)$ and expected $\left(H_{\mathrm{E}}\right)$ heterozygosities, and deviations from Hardy-Weinberg equilibrium (HWE) for each locus in the test population of $B$. schreberi were calculated using the program of GenAlex 6.5 (Peakall and Smouse, 2012).

\section{RESULTS AND DISCUSSION}

Twenty-eight of the 31 tested markers were polymorphic. The $N_{\mathrm{A}}$ ranged from 1 to 9 . The $H_{\mathrm{O}}$ and $H_{\mathrm{E}}$ ranged from 0 to 1 and from 0 to 0.751 , respectively (Table 1 ). All of the loci, except for locus BS29, showed significant deviations from HWE, which could be due to the extensive clonal reproduction of this species. These markers will facilitate further investigation into the adaptive genetic variation and conservation of $B$. schreberi.

Table 1. Primer sequences and characteristics of the 31 microsatellite loci in Brasenia schreberi.

\begin{tabular}{|c|c|c|c|c|c|c|c|c|}
\hline Locus & Primer sequence $\left(5^{\prime}-3^{\prime}\right)$ & Repeat motif & Allele size (bp) & $\mathrm{Ta}\left({ }^{\circ} \mathrm{C}\right)$ & $N_{\mathrm{A}}$ & $H_{0}$ & $H_{\mathrm{E}}$ & $\begin{array}{c}\text { GenBank } \\
\text { accession No. }\end{array}$ \\
\hline BS1 & $\begin{array}{l}\text { GAACGAATGAATAACCAGAGATG } \\
\text { ATTTCCTAGCCTGCCTCCAT }\end{array}$ & $(\mathrm{TG})_{7}(\mathrm{AG})_{8}$ & $127-138$ & 58 & 4 & 0.125 & 0.721 & KT899583 \\
\hline BS2 & $\begin{array}{l}\text { TTGTAGAAGTGCCACGATGC } \\
\text { CGCTTCCATTCTCTACCCAG }\end{array}$ & $(\mathrm{CT})_{9}(\mathrm{CA})_{7}$ & $147-159$ & 59 & 6 & 0.250 & 0.594 & KT899584 \\
\hline BS3 & $\begin{array}{l}\text { TCAAAACGTGAGCATGAACAA } \\
\text { TAACCATTCAAGAAACCCCG }\end{array}$ & $(\mathrm{CT})_{6}(\mathrm{AT})_{6}$ & $115-130$ & 60 & 4 & 0.125 & 0.572 & KT899585 \\
\hline BS4 & $\begin{array}{l}\text { TGTAGCCGACCCCATCTAGT } \\
\text { GGTTTCCACGTAACACGTCA }\end{array}$ & $(\mathrm{GTT})_{5}$ & $128-212$ & 59 & 4 & 0.125 & 0.558 & KT899586 \\
\hline BS5 & $\begin{array}{l}\text { GGAACATGAGCAAGTGCAGA } \\
\text { TTGGCAAGTTATACCCTCCG }\end{array}$ & $(\mathrm{AG})_{10}$ & 273-291 & 60 & 3 & 0.125 & 0.525 & KT899587 \\
\hline BS6 & $\begin{array}{l}\text { ATCCCCGTGTCACACTAAGC } \\
\text { GGACCTGGGTCATATTAGGGA }\end{array}$ & $(\mathrm{TA})_{7}(\mathrm{GA})_{7}$ & $209-218$ & 60 & 4 & 0.375 & 0.537 & KT899588 \\
\hline BS7 & $\begin{array}{l}\text { GGAGTGCAATAGGCAAAAGC } \\
\text { TGTTTCATTTTACAAAACGTGGA }\end{array}$ & $(\mathrm{ATG})_{5}$ & 118-184 & 60 & 4 & 1.000 & 0.593 & KT899589 \\
\hline BS8 & $\begin{array}{l}\text { CCAAAGATTCACACATTGGAAA } \\
\text { CTATGAAGTCACCAGGTGCG }\end{array}$ & $(\mathrm{AG})_{10}$ & $138-160$ & 60 & 6 & 0.417 & 0.751 & KT899590 \\
\hline BS9 & $\begin{array}{l}\text { CTTGGAAAGAGGGCTTTGTG } \\
\text { TGAGGTGAATGATTTTTGCATC }\end{array}$ & $(\mathrm{AAG})_{5}$ & $265-297$ & 60 & 3 & 0.000 & 0.392 & KT899591 \\
\hline BS10 & $\begin{array}{l}\text { TAACAGGAGCCGACGCTAGT } \\
\text { GTTGTACCGAAGCCTGAAGC }\end{array}$ & $(\mathrm{CT})_{6}$ & $270-281$ & 60 & 2 & 0.000 & 0.080 & KT899592 \\
\hline BS11 & $\begin{array}{l}\text { CCACTAAGACTTCCGGTGCT } \\
\text { GCGGCATGCATTACTCCTAT }\end{array}$ & $(\text { GAGAG })_{5}$ & 274 & 59 & 1 & 0.000 & 0.000 & KT899593 \\
\hline BS12 & $\begin{array}{l}\text { GGGGCTTCATTTATTGGGAT } \\
\text { CTGCTTTTCCATGGCATTTC }\end{array}$ & $(\mathrm{TC})_{7}$ & $252-280$ & 60 & 4 & 0.000 & 0.451 & KT899594 \\
\hline BS13 & $\begin{array}{l}\text { AATGGTGAAGGAAAGCATGG } \\
\text { GTGTCACGCACGCAGATAGT }\end{array}$ & $(\mathrm{CA})_{6}$ & $267-281$ & 60 & 4 & 0.125 & 0.194 & KT899595 \\
\hline BS14 & $\begin{array}{l}\text { GTGTCACGCACGCAGATAGT } \\
\text { GCAAGCTCATTTTGCTTTCTT }\end{array}$ & $(\mathrm{TG})_{6}$ & 178 & 60 & 1 & 0.000 & 0.000 & KT899596 \\
\hline BS15 & $\begin{array}{l}\text { TCGAGGTTTTCCTCTGCCTA } \\
\text { CATTTGCATGATTGATCGCT }\end{array}$ & $(\mathrm{TC})_{6}$ & $231-268$ & 60 & 4 & 1.000 & 0.631 & KT899597 \\
\hline BS16 & $\begin{array}{l}\text { CCGAAGAAGAAGTTCCTCCC } \\
\text { CATCAGCTGCCTCATCTTCA }\end{array}$ & $(\mathrm{GAA})_{7}$ & 105-114 & 60 & 4 & 0.792 & 0.614 & KT899598 \\
\hline BS17 & $\begin{array}{l}\text { TCAGAGCGACAGTGAGGAGA } \\
\text { CCAAAATCAGCATGTGCATC }\end{array}$ & $(\mathrm{TGA})_{5}$ & 184 & 60 & 1 & 0.000 & 0.000 & KT899599 \\
\hline BS18 & $\begin{array}{l}\text { CAAATCCCACATGTCTCACG } \\
\text { GCTTTTGGAATCTGCCAAACTA }\end{array}$ & $(\mathrm{T})_{11}$ & 168-177 & 60 & 4 & 0.958 & 0.591 & KT899600 \\
\hline BS19 & $\begin{array}{l}\text { CTGCTGAAGGATTCTCGGAC } \\
\text { TGTACAGCACTTGCAGGTAAGAA }\end{array}$ & $(\mathrm{CTA})_{5}$ & 263-289 & 60 & 2 & 0.000 & 0.413 & KT899601 \\
\hline BS20 & $\begin{array}{l}\text { GCAAGGATGATGTTTGAGCA } \\
\text { GAAATATATTCATGATGTGGAGTATGA }\end{array}$ & $(\mathrm{TA})_{7}(\mathrm{TG})_{6}$ & $100-161$ & 60 & 5 & 1.000 & 0.639 & KT899602 \\
\hline BS21 & $\begin{array}{l}\text { AAGGCGGAAAAGAGGAAGAG } \\
\text { GAGGATGGCTGATTGTGGTT }\end{array}$ & $(\mathrm{TG})_{6}$ & $141-148$ & 60 & 2 & 0.000 & 0.497 & KT899603 \\
\hline BS22 & $\begin{array}{l}\text { AAGTATGCCCAACCAGCAAA } \\
\text { AAGCTAACCGTTTCATCTTTATCA }\end{array}$ & $(\mathrm{TG})_{7}(\mathrm{AG})_{9}$ & $218-231$ & 60 & 6 & 0.417 & 0.491 & KT899604 \\
\hline BS23 & $\begin{array}{l}\text { AATAGACACGACCGGTTTGG } \\
\text { AAACCAAACCAAATGACCCA }\end{array}$ & $(\mathrm{AAC})_{6}$ & 105-141 & 60 & 4 & 0.917 & 0.650 & KT899605 \\
\hline BS24 & $\begin{array}{l}\text { TCGATCTTTGAAATTCGATAGTTG } \\
\text { ATGCACCAAACCAAAACACA }\end{array}$ & $(\mathrm{CT})_{6}$ & $100-127$ & 59 & 4 & 0.042 & 0.322 & KT899606 \\
\hline BS25 & $\begin{array}{l}\text { AAACTCCACCAGATCAACCG } \\
\text { AAAAATGCCCCCTTCTCTGT }\end{array}$ & $(\mathrm{AG})_{7}$ & $186-288$ & 60 & 3 & 0.000 & 0.288 & KT899607 \\
\hline BS26 & $\begin{array}{l}\text { TCAAACGATAAAAACATTTGAAGAA } \\
\text { CGATCGGTCTGATCTTGGAT }\end{array}$ & $(\mathrm{TC})_{9}$ & $307-313$ & 59 & 5 & 0.250 & 0.360 & KT899608 \\
\hline BS27 & $\begin{array}{l}\text { AAACCTCTGGTACTTGGAGACTTG } \\
\text { TGCAGTTTGTCTTTTCCAAGG }\end{array}$ & $(\mathrm{AT})_{8}$ & 107-187 & 60. & 8 & 0.000 & 0.743 & KT899609 \\
\hline BS28 & $\begin{array}{l}\text { GGCCTAGATTCCTTGCTTCC } \\
\text { CGGCAATCATTTCTGTTTTT }\end{array}$ & $(\mathrm{AC})_{6}$ & $241-280$ & 60 & 5 & 0.708 & 0.655 & KT899610 \\
\hline BS29 & $\begin{array}{l}\text { AATCAGATCGGACCACGAAC } \\
\text { GCATGTTCTAGAGGAAAGAGCC }\end{array}$ & $(\mathrm{GT})_{6}$ & $230-270$ & 60 & 6 & 0.417 & 0.698 & KT899611 \\
\hline BS30 & $\begin{array}{l}\text { AGGATCGAGTTGTTTTCCCC } \\
\text { CGTGGTGTAAGGCCAAGAGA }\end{array}$ & $(\mathrm{AG})_{6}$ & $216-232$ & 60 & 4 & 0.667 & 0.575 & KT899612 \\
\hline BS31 & $\begin{array}{l}\text { ACCTAGTTTCTCACCGCCAG } \\
\text { GGAAGAGAAGGGAGATGAAGG }\end{array}$ & $(\mathrm{CT})_{10}$ & $132-175$ & 59 & 9 & 1.000 & 0.731 & KT899613 \\
\hline
\end{tabular}

$\mathrm{Ta}=$ annealing temperature; $N_{\mathrm{A}}=$ number of alleles observed; $H_{\mathrm{E}}=$ expected heterozygosity; $H_{\mathrm{O}}=$ observed heterozygosity. 


\section{Conflicts of interest}

The authors declare no conflict of interest.

\section{ACKNOWLEDGMENTS}

We thank Chun-Feng Yang for his kind assistance in the field work, and Zhi-Zhong Li and Zhi-Yuan Du for their help in the laboratory. Research supported by grants from the National Natural Science Foundation of China (\#31570220 and \#31200170) and the Open Funding Project of the Key Laboratory of Aquatic Botany and Watershed Ecology, Chinese Academy of Sciences.

\section{REFERENCES}

Chen WS, Zhao G, Jian SG and Wang ZF (2015). Development of microsatellite markers for Suriana maritima (Surianaceae) using next-generation sequencing technology. Genet. Mol. Res. 14: 14115-14118.http://dx.doi.org/10.4238/2015. October.29.31

Kim CY, Na HR and Choi HK (2008). Conservation genetics of endangered Brasenia schreberi based on RAPD and AFLP makers. J. Plant Biol. 51: 260-268. http://dx.doi.org/10.1007/BF03036125

Kim CY, Jung JD, Na HR, Kim SW, et al. (2012). Population genetic structure of the endangered Brasenia schreberi in South Korea based on nuclear ribosomal spacer and chloroplast DNA Sequences. J. Plant Biol. 55: 81-91. http://dx.doi. org/10.1007/s12374-011-9193-4

Lee HW, Choung HL, Roh TH, Kwon YH, et al. (2005). Categorization and Conservation of the Threatened Plant Species in Environmental Impact Assessment. Korea Environment Institute, Seoul.

Peakall R and Smouse PE (2012). GenAlEx 6.5: genetic analysis in Excel. Population genetic software for teaching and research - an update. Bioinformatics 28: 2537-2539. http://dx.doi.org/10.1093/bioinformatics/bts460

Thiel T, Michalek W, Varshney RK and Graner A (2003). Exploiting EST databases for the development and characterization of gene-derived SSR-markers in barley (Hordeum vulgare L.). Theor. Appl. Genet. 106: 411-422.

Yun YE, Yu JN, Nam GH, Ryu SA, et al. (2015). Next-generation sequencing identification and characterization of microsatellite markers in Aconitum austrokoreense Koidz., an endemic and endangered medicinal plant of Korea. Genet. Mol. Res. 14: 4812-4817. http://dx.doi.org/10.4238/2015.May.11.13

Zhang FQ, Lei SY, Gao QB, Khan G, et al. (2015). Isolation of microsatellite loci for Rhodiola alsia (Crassulaceae), an important ethno-medicinal herb endemic to the Qinghai-Tibetan plateau. Genet. Mol. Res. 14: 5266-5269. http://dx.doi. org/10.4238/2015.May.18.18

Zhang GF and Gao BQ (2008). Analysis on genetic diversity and genetic structure of Brasenia schreberi in Jiangsu and Zhejiang Provinces revealed by ISSR Markers. J. Lake Sci. 20: 662-668. http://dx.doi.org/10.18307/2008.0516 